\title{
Cuidados odontológicos na paralisia supranuclear progressiva: relato de caso
}

\section{Dental carein progressivesupranudear palsy. a casereport}

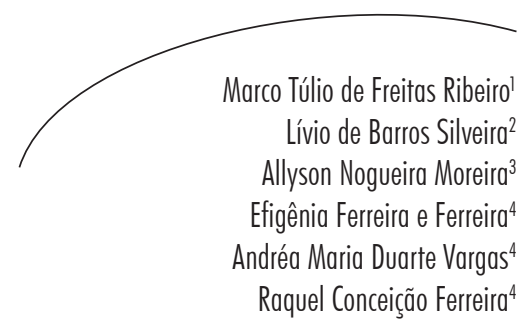

\section{Resumo}

Este trabalho teve como objetivo relatar o tratamento de complicações bucais numa paciente hospitalizada com diagnóstico de paralisia supranuclear progressiva (PSP), causa mais comum de parkinsonismo degenerativo. A paciente fazia uso de vários medicamentos, tinha 70 anos de idade, sem acompanhamento odontológico durante a hospitalização, com edentulismo total na arcada superior e usuária de prótese parcial na região inferior, mas em função do quadro clínico sistêmico não fazia uso da mesma. No exame bucal, constatou-se uma lesão na mucosa e lábio superior em decorrência de trauma dos dentes inferiores. Planejou-se inicialmente o tratamento da lesão com laserterapia e exodontias dos dentes que provocaram a lesão traumática. Após a cicatrização da lesão, os dentes inferiores remanescentes foram extraídos. Os resultados obtidos da terapêutica aplicada foram eficazes na melhora clínica da paciente. Concluise, assim, que é marcante a necessidade de um cirurgião-dentista como membro permanente da equipe multidisciplinar para o tratamento dessa e de outras patologias, a fim de permitir melhor qualidade de vida aos pacientes.

\section{Abstract}

This study aimed to report the treatment of oral complications in hospitalized patients diagnosed with progressive supranuclear palsy (PSP), the most common cause of degenerative parkinsonism. The patient was taking several medications, was 70 years old, without dental monitoring during hospitalization, with partial edentulism (without natural teeth in upper arch) and used a lower partial denture, but due to clinical history

\footnotetext{
Casa de Saúde Santa Izabel, Complexo de reabilitação e cuidado ao idoso. Fundação Hospitalar do Estado de Minas Gerais. Betim, MG, Brasil.

2 Escola de Engenharia, Departamento de Engenharia Mecânica. Universidade Federal de Minas Gerais. Belo Horizonte, MG, Brasil.

3 Faculdade de Odontologia, Departamento de Odontologia Restauradora. Universidade Federal de Minas Gerais. Belo Horizonte, MG, Brasil.

4 Faculdade de Odontologia, Departamento de Odontologia Social e Preventiva. Universidade Federal de Minas Gerais. Belo Horizonte, MG, Brasil.
}

Palavras-chave:

Administração de Caso. Idoso. Saúde Bucal. Lasers. Doença de Parkinson. 
she was not using it. On examination, there was an oral mucosal damage and upper lip due to trauma of the lower teeth. It was planned to initially treat the lesion with laser therapy and extractions of teeth that caused the traumatic injury. After healing of the injury, the remaining lower teeth were extracted. The results of the treatment applied were effective in improving the clinical condition of the patient. This case report showed the need for a dentist as permanent member of the multidisciplinary team to treat this type and other conditions to allow better quality of life for patients.
Key words: Case

Management. Aged. Oral

Health. Parkinson Disease,

Lasers.

\section{INTRODUÇÃO}

O envelhecimento populacional é acompanhado pelo aumento da prevalência de doenças crônico-degenerativas, ${ }^{1}$ podendo acarretar comprometimento e incapacidade funcional. A dependência para o desempenho das atividades de vida diária tende a aumentar de cerca de 5\% na faixa etária de 60 anos para, aproximadamente, $50 \%$ entre os com 90 anos ou mais. $^{2}$

A paralisia supranuclear progressiva (PSP), também chamada síndrome Steele-RichardsonOlszenskei, é uma síndrome neurodegenerativa distinta e, provavelmente, subdiagnosticada. É a causa mais comum de parkisionismo degenerativo depois da síndrome de Parkinson. ${ }^{3,4}$ Patologicamente, a PSP é definida pelo acúmulo da proteína Tau, ${ }^{5}$ acúmulo de emaranhados neurofibrilares no núcleo subtalâmico, globo pálido, núcleo rubro, substância negra, estriado, tegumento da ponte, núcleos oculomotores, bulbo e núcleo denteado. ${ }^{4} \mathrm{O}$ quadro clínico se caracteriza por oftalmoparesia supranuclear, instabilidade postural e demência. ${ }^{4-6}$

A prevalência dessa doença é dependente da idade e estimada em 6-7 casos por 100.000 habitantes, com pico de início na idade de 63 anos, sendo que nenhum caso foi diagnosticado antes dos 40 anos de idade. ${ }^{4} \mathrm{O}$ diagnóstico correto é normalmente feito de 3,6 a 4,9 anos após o início dos sinais clínicos. ${ }^{5}$

Com a evolução da doença, os pacientes podem apresentar complicações como quedas, disfagia, problemas nutricionais, pneumonia aspiratória, redução na capacidade de piscar, paralisia vertical, salivação excessiva e apraxia da abertura palpebral, dentre outras. ${ }^{4}$ Esse quadro demanda cuidados de uma equipe multidisciplinar de saúde, que é essencial no manejo do paciente com PSP. A literatura não explicita a participação do cirurgiãodentista nessa equipe, ${ }^{7}$ mas dadas as complicações descritas, esse profissional pode contribuir para melhorar a qualidade de vida desses pacientes.

Neste trabalho será relatado um caso clínico de uma paciente portadora de PSP, cuja rigidez muscular comprometeu o uso da prótese dentária superior, com consequente perda da dimensão vertical de oclusão e formação de uma lesão ulcerativa traumática nos tecidos intra e periorais; que foi tratada com laserterapia.

\section{RELATO DO CASO CLÍNICO}

Paciente do sexo feminino, feoderma, viúva, 70 anos de idade, foi admitida em uma instituição privada de longa permanência (ILP) do município de Belo Horizonte, com PSP, hipotireoidismo, hipertensão e quadro depressivo. Paciente fazia uso de fármacos antiparkisonianos (Levodopa + Benserazida), hormônios (Levotiroxina sódica), anti-hipertensivos (Hidroclorotiazida, Enalapril) e antidepressivos (Cipramil ou Citalopran). Apresentava comprometimento cognitivo leve, mantendo a compreensão e a resposta aos comandos verbais.

Ao exame odontológico, quando da admissão na ILP, a paciente usava uma prótese parcial 
removível (PPR) superior apoiada nos dois primeiros molares superiores remanescentes (figura 1). Na região inferior, a paciente possuía os pré-molares, incisivos e caninos. A condição de saúde bucal foi considerada satisfatória nesse momento, com ausência de lesões cariosas, doença periodontal e alterações de mucosa bucal, não sendo indicado nenhum tratamento odontológico. O cirurgião-dentista recomendou a manutenção do uso da prótese, uma vez que esta foi considerada satisfatória e o seu uso bem suportado pela paciente.

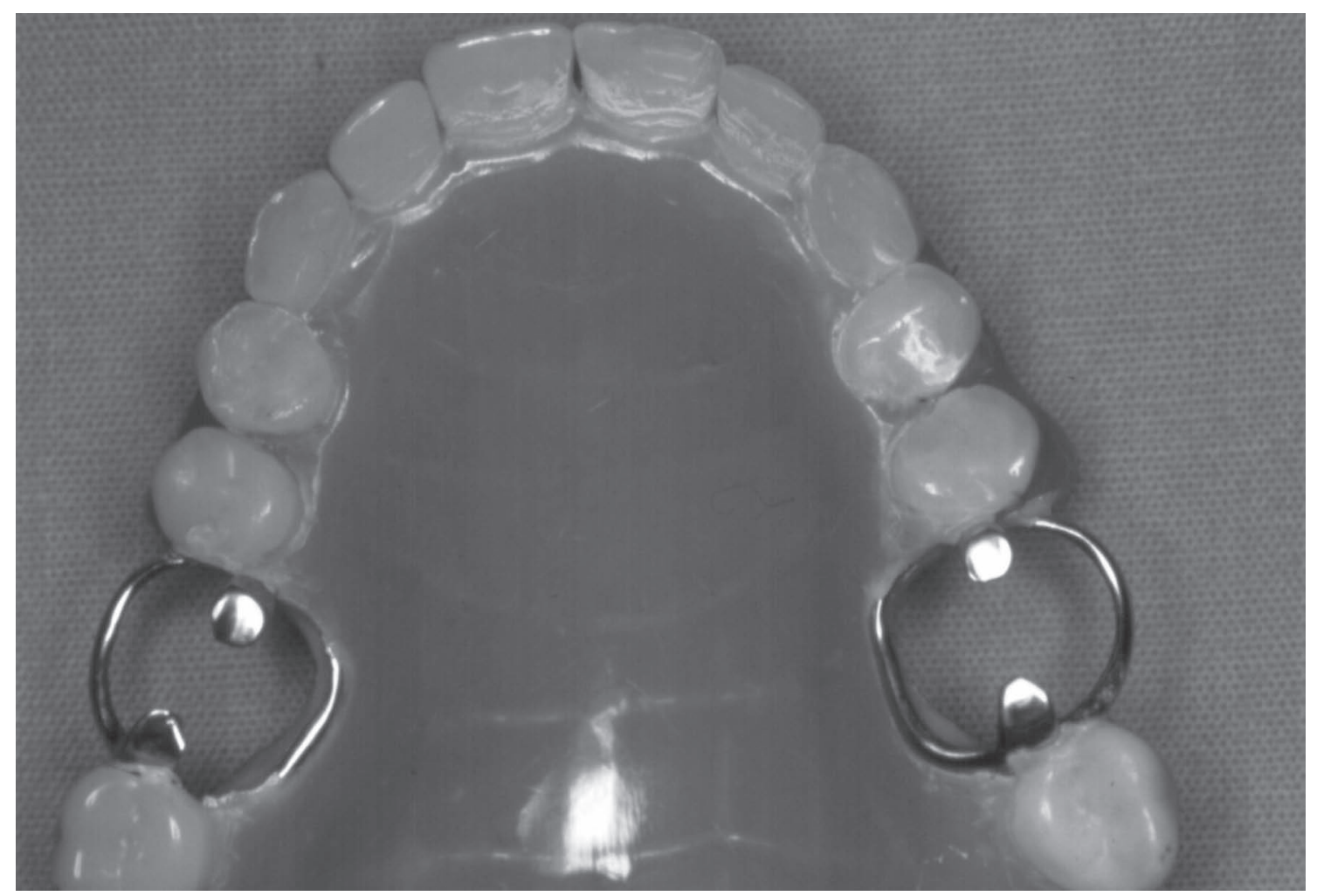

Figura 1 - Prótese parcial removível superior utilizada pela paciente até a sua hospitalização.

Aproximadamente nove meses após sua admissão na ILP, a paciente apresentou uma piora da condição sistêmica, levando à sua hospitalização. Durante o período de internação hospitalar, a paciente não fez uso da PPR. Na ocasião os médicos relataram que, devido à rigidez muscular severa dos músculos da face, a prótese estava traumatizando a mucosa bucal. Apesar da orientação do cirurgião-dentista quanto ao risco de desenvolvimento de lesões ulcerativas traumáticas sem o uso da prótese, em função da oclusão dos dentes naturais inferiores contra o rebordo alveolar superior, os médicos mantiveram esta conduta.
Como previsto pelo cirurgião-dentista, ocorreu uma lesão ulcerativa traumática no lábio superior de $1,8 \mathrm{~cm}$, assim como na região anterior do rebordo alveolar superior (figuras 2 e 3). Esta lesão resultou da oclusão dos incisivos inferiores sobre o lábio e mucosa mastigatória pela falta de contatos oclusais entre dentes posteriores inferiores e superiores. Tal situação pode ter sido agravada pelas irregularidades nas incisais dos dentes inferiores. Nesse momento, a paciente apresentava fácies de dor, supostamente associada com a lesão descrita. 


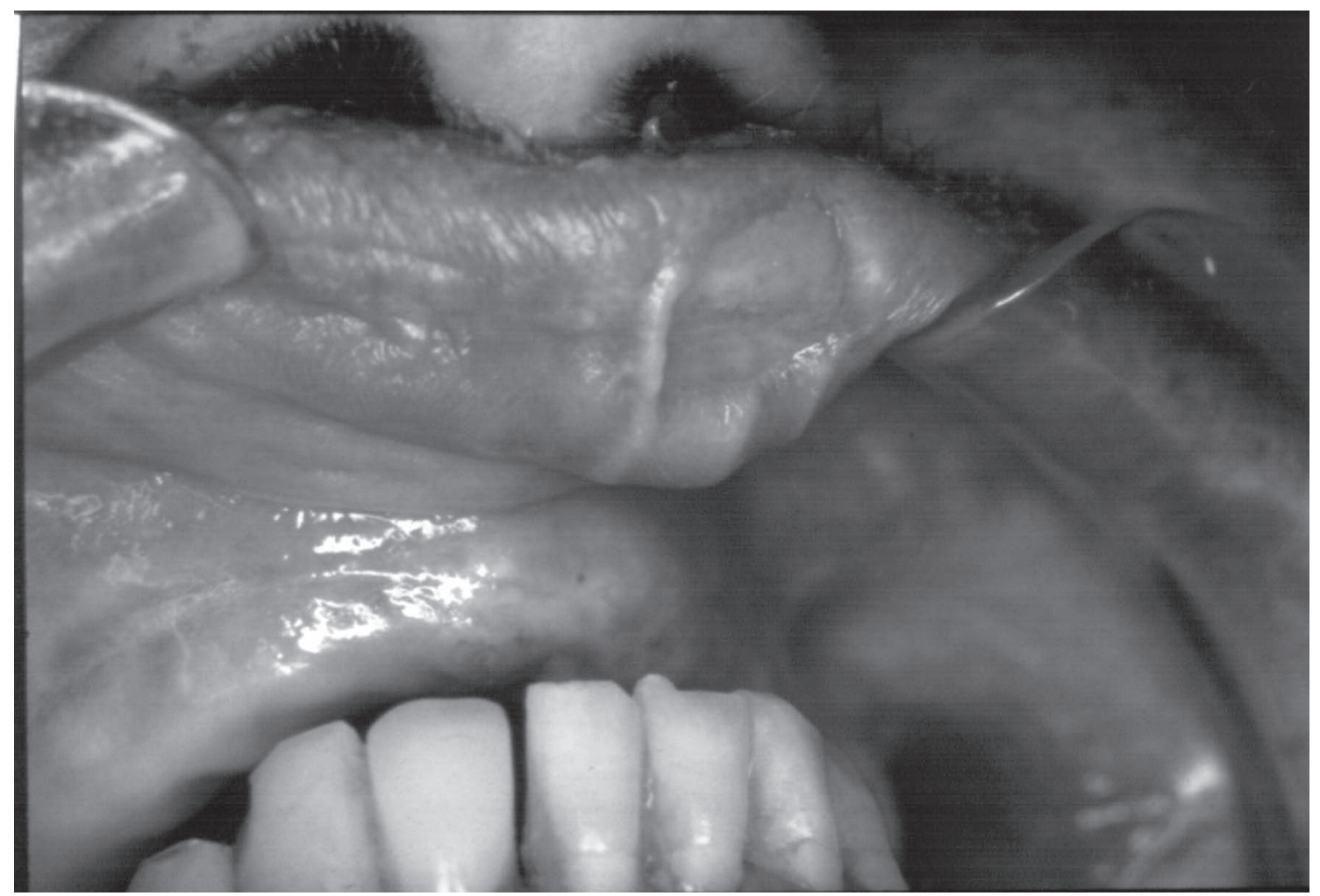

Figura 2 - PB Lesão ulcerativa no rebordo alveolar superior.

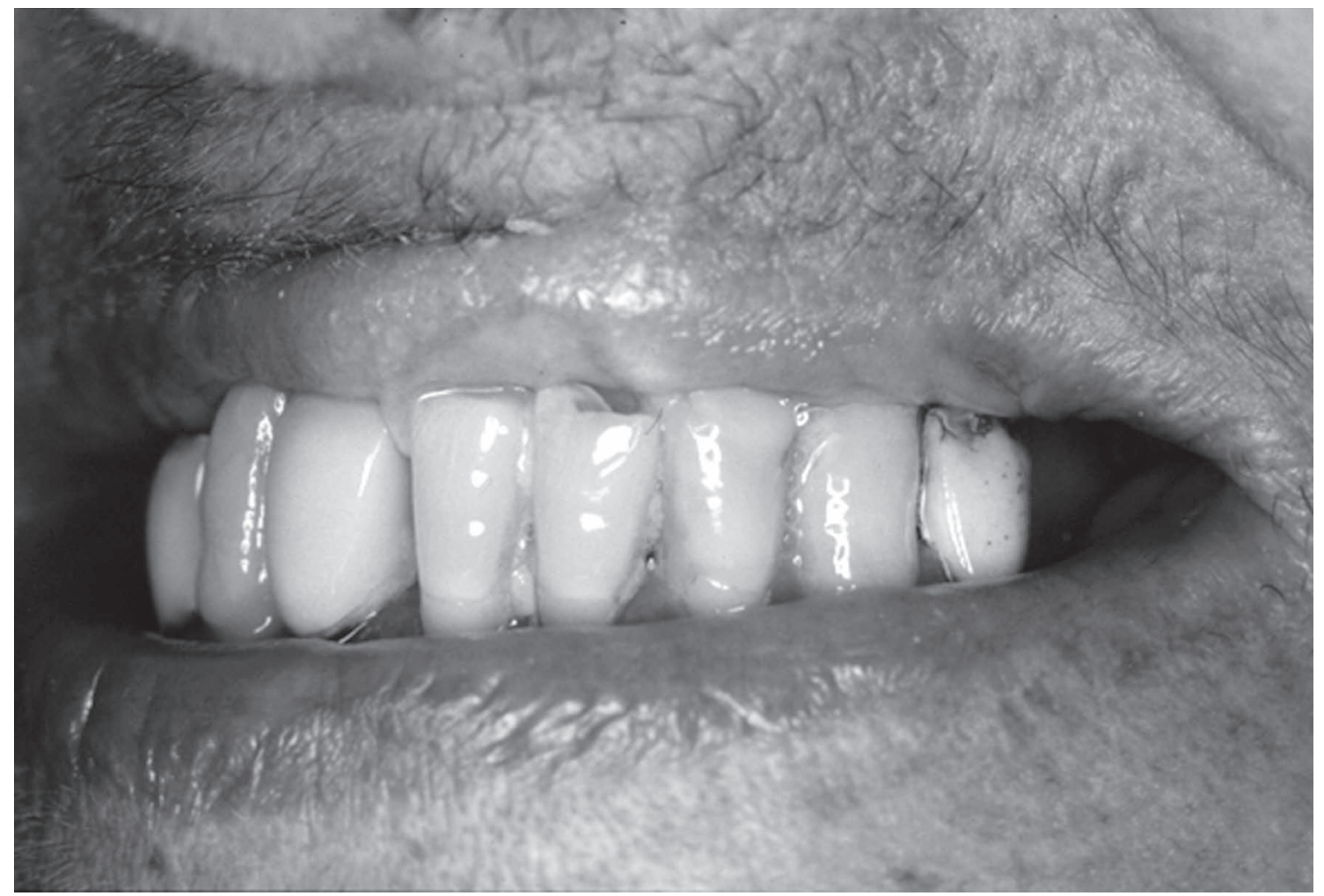

Figura 3 - PB Lesão ulcerativa no lábio superior na região de incisivos inferiores. 
Diante desse quadro, o cirurgião-dentista indicou a extração imediata somente dos elementos diretamente associados ao trauma, para permitir a regressão da lesão. A manutenção dos dentes com a recolocação da prótese foi considerada inviável, devido a grande limitação de abertura de boca e rigidez muscular, além do uso de sonda para alimentação.

No entanto, em função de problemas na autorização para as extrações e persistência de fácies de dor pela paciente, foi proposto tratamento laserterápico para as lesões ulcerativas até que a autorização fosse obtida para remoção dos outros dentes. Foi empregado um laser diodo de baixa intensidade DR 500 LaserBeam, com o seguinte protocolo de aplicação: comprimento de onda vermelho $(\lambda=685 \mathrm{~nm})$, modo pulsado (36 Hertz), potência de $50 \mathrm{mWatts}$, durante dois minutos e meio, resultando numa energia depositada de 7,5 Joules/ponto e dose aproximada de 187 Joules $/ \mathrm{cm}^{2}$. Foram realizadas aplicações diárias sobre um único ponto centralizado na lesão por um período de sete dias, ocorrendo a regressão satisfatória da lesão durante esse período (figuras 4 e 5) e melhora da fácies de dor.

Após autorização pelo curador, todos os dentes remanescentes foram extraídos, evitandose a recidiva das lesões traumáticas.

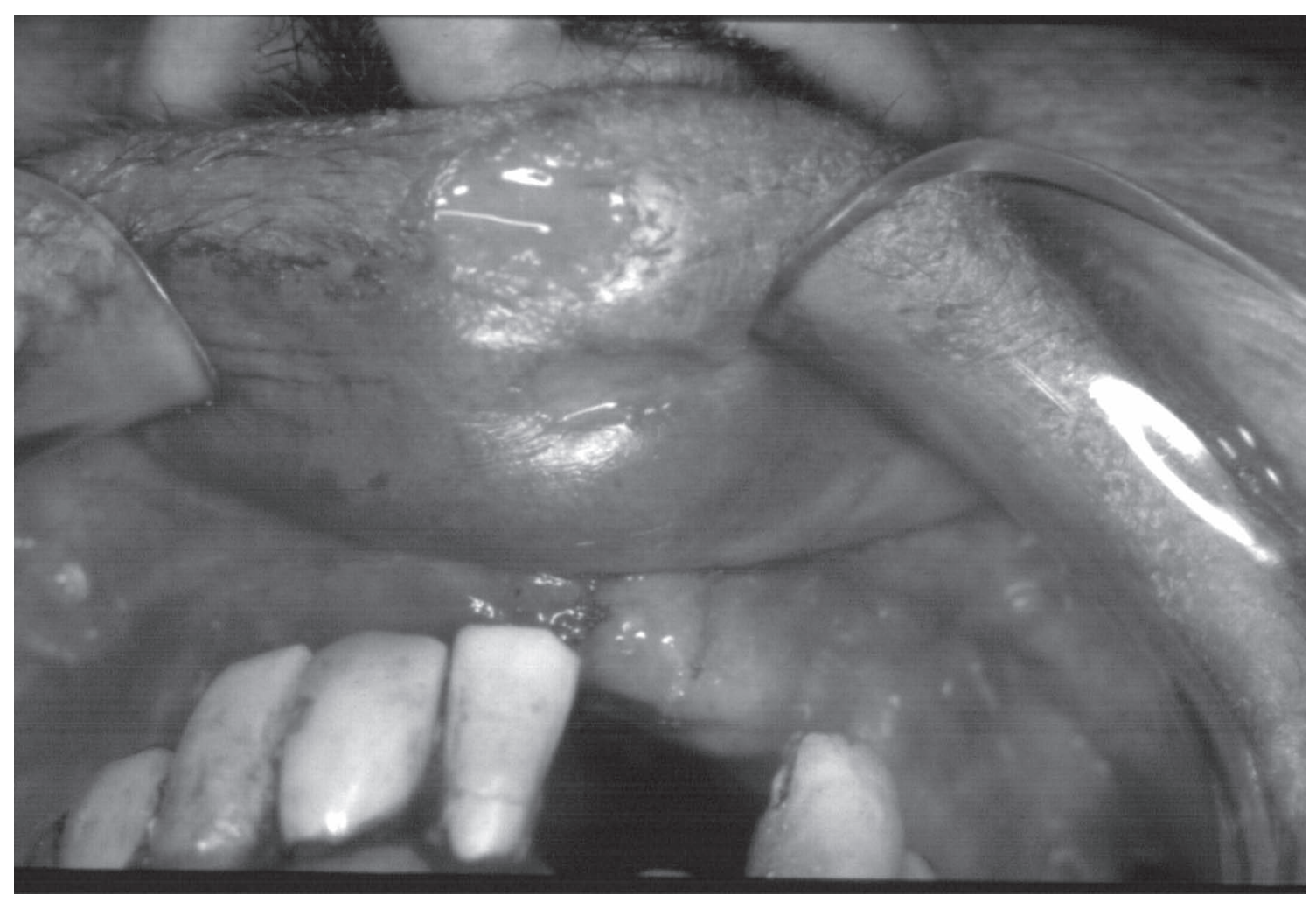

Figura 4 - PB Aspecto da lesão no lábio superior no momento da extração do incisivo inferior. 


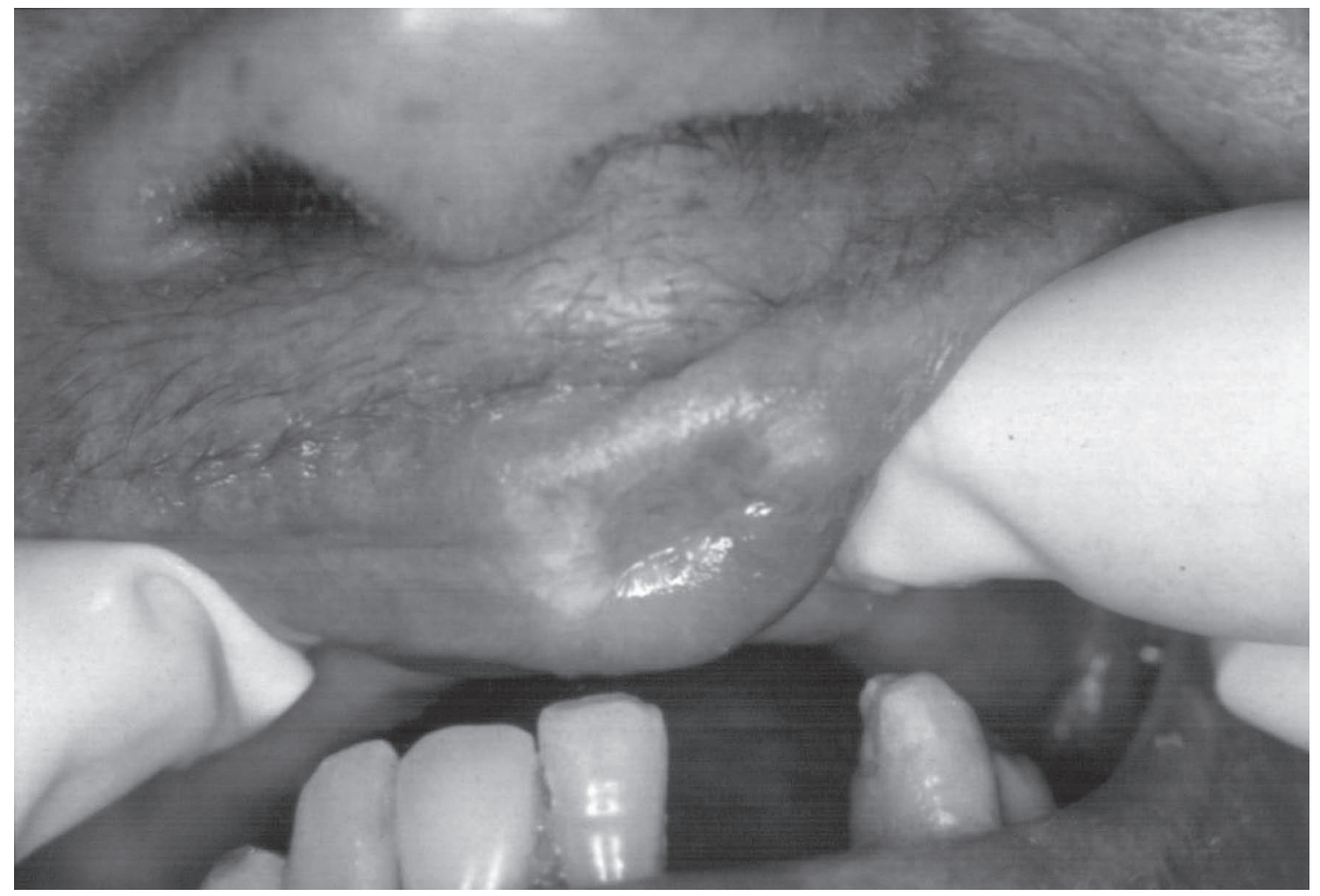

Figura 5 - PB Aspecto da lesão no lábio superior após a extração do incisivo inferior e as sessões de laserterapia.

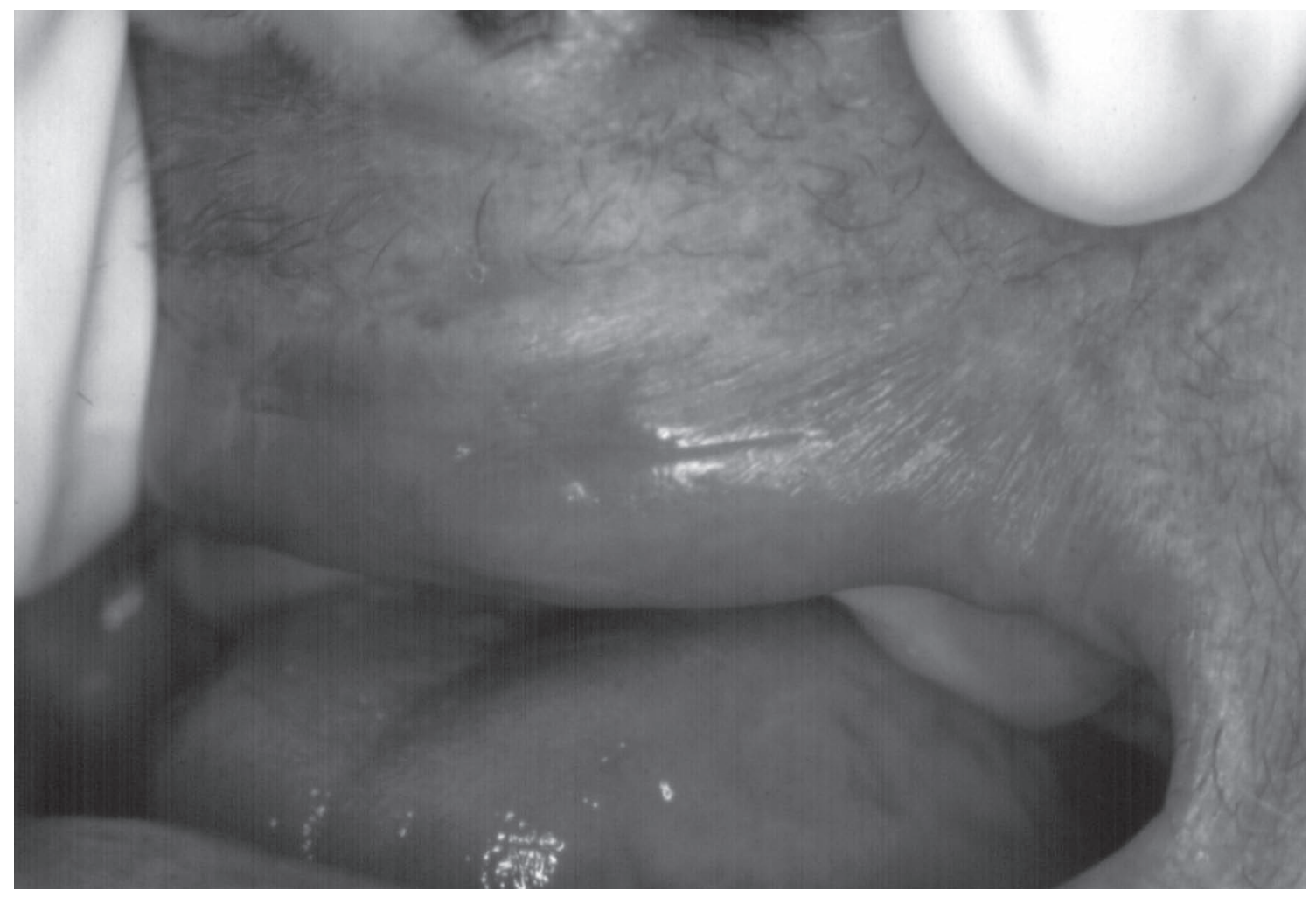

Figura 6 - Aspecto final da lesão do lábio após as extrações dos dentes remanescentes. 


\section{DISCUSSÃO}

O relato desse caso mostra a importância da equipe interdisciplinar nos cuidados aos pacientes com PSP e a relevância da Odontologia, quer seja no diagnóstico ou no tratamento das complicações bucais da PSP. A literatura, ao descrever os profissionais envolvidos nesses cuidados, sequer cita o cirurgião-dentista.

A ausência de cirurgiões-dentistas nas equipes de cuidados paliativos a estes pacientes leva a decisões sem o devido suporte técnico/ científico, gerando muitas vezes complicações em sua evolução. Este caso ilustra com clareza tais constatações, uma vez que a participação do cirurgião-dentista por parte da equipe ocorreu somente após determinação médica de remoção da PPR e da lesão instalada, ainda que a paciente recebesse atenção contínua do profissional na ILP. A atenção odontológica deve ocorrer o mais precocemente possível, adotando-se uma abordagem interdisciplinar. A PSP é uma patologia sem perspectiva de cura e a equipe de profissionais envolvidos no cuidado a esses pacientes devem conhecê-la, bem como suas complicações, possibilidades de manejo e tratamento, propiciando alívio de seus sintomas.

O laser de baixa intensidade foi a terapia escolhida por promover alívio da dor (efeito antiálgico), reparação tecidual (efeito bioestimulante do tropismo celular), redução de edema, da hiperemia (efeito antiinflamatório e normalizador circulatório) e da dor. $^{8-11} \mathrm{~A}$ terapia laser de baixa intensidade provoca aumento da microcirculação e neovascularização sobre a superfície do ferimento, redução do microedema que ocorre nos tecidos subjacentes (com a melhoria do fluxo sanguíneo), aumento significativo do tecido de granulação, da atividade, bem como número de fibroblastos, crescimento do número e atividade de mastócitos e macrófagos e significativo estímulo da reepitelização das margens da ferida. ${ }^{9}$ Walsh,${ }^{11}$ em sua revisão sobre 30 anos de experiência com laser de baixa intensidade e bioestimulação em Odontologia, relatou que esta terapia estimula a proliferação fibroblástica, ativa fagocitose nas células do sistema imune, provoca quimiotaxia para leucócitos, direciona e seleciona o sistema imune, aumenta a produção de queratina e a mobilidade dos queratinócitos, e ativa a proliferação, diferenciação e calcificação dos osteoblastos.

$\mathrm{Na}$ adoção da laserterapia, deve-se definir o tipo de laser, a dosimetria e a quantidade de energia a ser aplicada sobre determinado tecido, ${ }^{12-15} \mathrm{O}$ protocolo de tratamento adotado neste caso seguiu as recomendações preconizadas na literatura ${ }^{9,10,16}$ e mostrou-se eficaz na cicatrização da lesão ulcerada.

$\mathrm{Na}$ perspectiva do cuidado paliativo, o tratamento desse caso mostrou que a participação do cirurgião-dentista trouxe alívio da dor, por meio de uma terapia conservadora e com resultados imediatos. Vale ressaltar que, apesar da comprovada eficiência da laserterapia ${ }^{8-12}$ e do seu uso potencial nos cuidados paliativos, essa técnica ainda é, de modo geral, pouco difundida e disponibilizada para a população.

Este trabalho contribui também na difusão dos benefícios potenciais da laserterapia na atenção à saúde bucal dos indivíduos com doenças crônicas degenerativas, como a PSP.

\section{CONCLUSÕES}

Nesse caso, a atuação do cirurgião-dentista foi necessária para resolução das complicações bucais decorrentes da PSP, evidenciando a importância da abordagem interdisciplinar no alívio dos seus sintomas. Os cuidados paliativos exigem da equipe de saúde conhecimento de novas terapias das diversas áreas, bem como grande sensibilidade na escolha daquela mais indicada à condição do paciente. 


\section{REFERÊNCIAS}

1. Lima-Costa MF. Epidemiologia do envelhecimento no Brasil. In: Roiquayrol MZ, Almeida Filho N. Epidemiologia \& Saúde. Rio de Janeiro: Medsi; 2003. p. 499-513.

2. Brasil. Ministério da Saúde Cadernos de atenção básica: envelhecimento e saúde da pessoa idosa. Brasília: Ministério da Saúde; 2007.

3. Steele JC, Richardson JC, Olszewski J. Progressive supranuclear palsy. A heterogeneous degeneration involving the brain stem, basal ganglia and cerebellum with vertical gaze and pseudobulbar palsy, nuchal dystonia and dementia. Arch Neurol 1964; 10: 333-59.

4. Barsottini OG, Felício AC, Aquino CC, Pedroso JL. Progressive supranuclear palsy: new concepts. Arq Neuropsiquiatr. 2010 Dec;68(6):938-46.

5. Willians DR, Lees AJ. Progressive supranuclear palsy: clinicopathological conceps and diagnostic challenges. Lancet Neurol 2009; 8(3): 270-9.

6. Santacurz P, Uttl B, Litvan I, Grafman J. Progressive supranuclear palsy: a survey of disease course. Neurology 1998; 50 (6): 1637-47.

7. Burn DJ, Warren NM. Toward future therapies in progressive supranuclear palsy. Mov Disord 2005; 20(Suppl 12): S92-S8.
8. Lopes LA, Brugnera AAJR. Aplicações clínicas do laser não cirúrgico. In: Brugnera AAJR, Pinheiro AL. Lasers na Odontologia moderna. São Paulo: Pancast; 1998. p. 99-120.

9. Simunovic Z, Ivankovich AD, Depolo A. Wound healing of animal body sport traffic accident using low-level laser therapy treament: a randomized clinical study of seventy-four patients with control group. J Clin Laser Med Surg 2000; 18(2): 67-73.

10. Mello J B, Mello GPS, Mello LS. Laser de baixa potência In: Mello JB, Mello GPS. Laser em odontologia. São Paulo: Santos; 2001. p. 71-85.

11. Walsh LJ. The current status of low laser therapy in dentistry. Part 1. Soft tissue applications. Aust Dent J 1997; 42(4): 247-54.

12. Wolbarsht ML. Laser applications in medicine and biology. New York: Plenum Press; 1991.

13. Goldman L. The biomedical laser: technology and clinical applications. New York: Springer Verlag; 1981.

14. Pimenta LHM. Laser em medicina e biologia. São Paulo: Roca; 1990.

15. Genovese WJ. Laser de Baixa intensidade: aplicações terapêuticas em Odontologia. São Paulo: Santos; 2007.

16. Neiburger EJ. The effect of low-power lasers on intraoral wound healing. N Y State Dent J 1995; 61(3): 40-3. 